\title{
Antipurinergic Therapy with Suramin as a Treatment for Autism Spectrum Disorder
}

\author{
Rafie Hamidpour ${ }^{*}$, Soheila Hamidpour², Mohsen Hamidpour ${ }^{2}$, Marriam Zarabi ${ }^{4}$, Mahnaz Sohraby ${ }^{4}$, \\ Mina Shalari ${ }^{4}$ \\ ${ }^{1}$ Department of Herbal Medicine, Pars Bioscience, Leawood, Kansas, USA \\ ${ }^{2}$ School of Medicine Department of Pathology, University of Kansas City, Missouri, USA \\ ${ }^{3}$ Department of Hematology and Blood banking-Faculty of Paramedical Sciences- Shahid Beheshti University of Medical Sciences-Tehran, Iran \\ ${ }^{4}$ Pars Bioscience, Leawood, Kansas, USA
}

*Corresponding author: Dr. Rafie Hamidpour, Pars Bioscience, LLC, 14109 Cambridge Lane, Leawood, Kansas 66224, USA, Tel: (913) 432-0107; Fax (913) 432-5708; E-mail: rafie@parsbioscience.com

Rec Date: Jan 08, 2016; Acc Date: Mar 24, 2016; Pub Date: Mar 29, 2016

Copyright: ( $)$ 2016, Hamidpour R, et al. This is an open-access article distributed under the terms of the Creative Commons Attribution License, which permits unrestricted use, distribution, and reproduction in any medium, provided the original author and source are credited.

Citation: Hamidpour R, Hamidpour S, Hamidpour M, et al. Antipurinergic Therapy with Suramin as a Treatment for Autism Spectrum Disorder. $J$ Biomedical Sci. 2016, 5:2.

\section{Abstract}

This review article discusses the opportunities and possibilities of antipurinergic therapy (APT) with suramin on mice models with ASD and fragile $X$ syndrome. Suramin is an APT mediator that triggers the mechanism linked to mitochondria, influences immunity, and has the ability to stabilize locomotor function and coordination, social behavior, normalize brain synapse structure, cell-to-cell signaling, and recover mitochondrial metabolism in mice with autistic-like behaviors and genetics.

Suramin may provide new therapeutic strategies for ASD subtypes. Many clinical studies have explored the scope of APT with suramin to assist with the genetic, metabolic, and environmental risk factors of ASD. The pharmacological components of suramin allow binding against purinergic receptors without release, serving as an antagonist of extracellular APT, and other nucleotides.

In recent studies, APT and suramin have been investigated to help correct behavior, genetic irregularities, and metabolism stemmed from neurodevelopmental disorders. This review article looks at APT with suramin under the following conditions in animal models:

- Gene-environment interaction

- $\quad$ APT with poly(I:C) mouse model

- Fragile $X$ mental retardation syndrome 1 (Fmr1) knockout mouse model

This review article investigates studies by Naviaux et al. and the influence of suramin and APT in relation to ASD and fragile $X$ syndrome. It was concluded that APT with suramin assists in correcting genetic abnormalities and environmental predispositions that may impact social behavior related to ASD.

Keywords: Antipurinergic therapy; Autism spectrum disorder; Fmr1; Fragile $\mathrm{x}$ syndrome; Maternal immune activation; Poly(I:C); Purinergic; Suramin

\section{List of Abbreviations}

ASD: Autism Spectrum Disorders; APT: Antipurinergic Therapy: APT; Fmr1: Fragile X Mental Retardation Genelocus 1; P2Y: GProtein Coupled Purinergic Receptors; P2X: Lonotropic Purinergic Receptors; MIA: Maternal Immune Activation; Poly(l:C): Polyinosinic

\section{Background}

This review article discusses the opportunities and possibilities of suramin on mice models with ASD and fragile $X$ syndrome. According to the CDC's Autism and Developmental Disabilities Monitoring (ADDM) Network, 1-2\% of children in the United States are affected by ASD [1]. Behaviors of ASD, as described by the ADDM Network, include limited social behavior and communication, repetitive movements, limited and heightened interest in selective activities and interests, including preferences related to taste, color, clothing, and social activities [1]. These preferences range from high-functioning to functioning, and independent to non-independent [2]. Genetic risk factors include various metabolic, environmental and genetic conditions, although these factors influence each child differently [1].

The objective of this review article is to determine if controlled doses of suramin alleviate ASD conditions, taking a specific look at clinical studies and research conducted by Naviaux et al. This review article includes findings by Naviaux et 
al. that discuss suramin and the relationship between MIA mouse model and poly $(I: C)$ and the effect signaling molecules of the mitochondria [2-5]; MIA and fragile $X$ models, as related to genetic impact of ASD [6]. It can be concluded that suramin doses had a positive effect on ASD and other neurodevelopment disorders.

Content for this review article was gathered using search terms "suramin and autism;" "suramin therapy and autism;" "autism therapy;" and "autism," through public electronic databases such as ProQuest, PubMed, Sciencedirect, and Google Scholar. Publications included Molecular Autism, Clinical Therapeutics, and PLOS ONE. Exclusion and inclusion of criteria included clinical studies tested alternative therapeutic methods using APT with suramin to repair genetic abnormalities as related to neurodevelopmental disorders, including ASD, and fragile $X$ syndrome. Research included studies and research that supported recent clinical studies conducted by Naviaux et al. on autism drug treatment. The following gene strains on mouse models found APT with suramin to repair damaged synapses and improve behavior:

\section{- $\quad$ APT with polyinosinic:polycytidylic acid (poly(l:C)) mouse model}

\section{- $\quad F V B$ and Fragile X (Fmr1) knockout mouse mode}

Controlled doses of suramin were found to repair damaged synapse and improve behavior related to ASD.

Tests with fragile $X$ mouse model found that APT with suramin positively influenced the synapse structural irregularities, metabolism, and behavior. APT with suramin also showed positive results in environmental MIA model. APT provides new research opportunities into pathogenesis and new drug development for human ASD and other spectrum disorders. The repairing methods and capabilities of APT with suramin can be better understood with additional clinical studies. Recommendations for future research include clinical and preclinical trials on suramin as a novel therapy.

\section{Review}

\section{APT}

APT is a nucleoside triphosphate that moves chemical energy from cell to cell and assists with metabolism production. Comprised of a purine base, APT is a significant player in metabolic processes. APT is also responsible for extracellular and intracellular signaling. The majority of APT synthesis takes place in the mitochondria [2]. Combination APT and suramin determine suramin's ability to alter purinergic activity and purine metabolism [3-5]. This is significant because purinergic triggers change under metabolic conditions and purine metabolism is a key translator for biochemicals altered by various treatments, including treatments in the maternal immune activation mouse model (MIA) mouse model of ASD [3-6].

Suramin may provide new therapeutic strategies for ASD subtypes [7]. Many clinical studies have explored the scope of APT with suramin to assist with the genetic, metabolic, and environmental risk factors of ASD. The pharmacological components of suramin allow binding against purinergic receptors without releasing it, serving as an antagonist of extracellular APT, and other nucleotides. Suramin has the strongest impact on purine metabolism [3-5].

In recent studies, APT and suramin have been investigated to help correct behavior, genetic irregularities, and metabolism stemmed from neurodevelopmental disorders. A large portion of this review article looks at studies by Naviaux et al., the analysis of suramin and APT, and opportunities for suramin therapy. It was concluded that APT with suramin does assist in correcting genetic abnormalities and environmental predispositions that may impact social behavior related to ASD.

\section{Gene-environment Interaction}

A variety of genetic risk factors have been identified that link rare mutations with ASD. Using genome-wide techniques and sequence-based tools assist in identifying the influence of rare genetic mutations and ASD, the effect of rare mutations and ASD is better understood. The Autism Genome Project genome-wide association conducted a study investigating the influence of common factors that may increase the risk for ASD, called the genome-wide association study (GWAS) [7]. The Autism Genome Project studied the genomic regions of nucleotide polymorphisms of approximately 1,400 families, including an analysis of genetic family history. The GWAS found a connection of SNP rs4141463 in MACROD2, and an SNP at 5P14.1 and 5P15.2 [7]. Previously, the GWAS did not find a similar link when studying nucleotide polymorphisms. In a similar study of 1,170 individuals of European heritage with ASD and 35307 controls (without ASD), there was no link between the MACROD2 identifier and rs4141463 [7]. To develop an inclusive analysis of ASD risk factors, approximately 1,301 families with ASD and their nucleotide polymorphisms were studied. Using an allele-score method to records the individual's number of alleles and associated alleles helped determine the variance level of ASD and the genomic regions of nucleotide polymorphisms [8]. The Autism Genome Project genome-wide association studied a total of 2,705 families to assess the individual single nucleotide polymorphisms and other common denominators to better understand the risk factors of ASD, but no single nucleotide polymorphism was identified in relation to ASD [8]. The identified single nucleotide polymorphism found was rs1718101 in a gene called CNTNAP2 [9]. This gene is vulnerable for ASD.

Similarly, a study determining the variances in genes of patients with ASD looked at the genotype arrays by comparing 996 individuals with ASD of European decent to 1,287 individuals (controls) [9]. Although ASD is known to be heritable, Pinto et al. aimed to find genic copy number variants and how this affects other genetic causes of ASD. A high number of unique, genic copy number variants were found de novo and inherited events, respectively, among a single family [9]. This finding complicates ASD genes SHANK2, SYNGAP1, DLGAP2 and DDX53-PTCHD1 [9]. This finding may provide a useful link to understanding the complex connected genetic pathways of ASD. 
Casey et al. used homozygous haplotype mapping to determine the genomic variation as the genetic influence of ASD as novel studies have suggested copy number variation as the causation of the genetic influence of ASD [10]. Using homozygous haplotype mapping to explore how and why identical haplotype structures are more common in individuals with ASD compared to individuals without ASD (controls). The study was conducted on 1,402 Autism Genome Project trios, respectively, and 1 million single nucleotide polymorphisms were coded. Findings included 1,218 new genes that may be linked to ASD, and 25 known genes that are linked to ASD. This finding supports the idea that homozygous haplotype regions have genes that linked to ASD and the significance of homozygous haplotype mapping to achieve comprehensive genome-wide data [10].

Genetic predisposition can also identify the possible cause and effect of internal and external factors that may influence gene-environment relations that impact the clinical results of patients with ASD.

Using a method of DNA polymorphism analysis to categorize the different causes and influences in ASD subtypes, Lao investigates genomic analysis as a comprehensive solution for ASD and other neurodevelopment disorders [7].

Lao looked at three genetic pathways to better understand the prognostic significance for individuals with ASD. Mixed sequences of these pathways determine the clinical result of a patient and related medical conditions. Accordingly, the three pathways include:

1. Genetic factors associated with the immune system and inflammatory responses related to ASD pathogenesis;

2. Genetic factors that impact the behavior and function of the main cellular defense tools that assist in reversing oxidative stress;

\section{Pharmacogenetics and doses of medical prescription.}

Identifying the environmental and genetic causes of a patient with ASD is difficult due to non-determined environmental conditions that may alter the genetic function and capabilities over time. The genetic test proposed by Lao suggests ASD diagnoses based on environmental and genetic factors. By analyzing the neuroplasticity potential and prognosis modifiers, and gaining a better understanding of the nine different clinical subtypes of ASD, a comprehensive treatment plan can be designed specific to each patient and patient needs.

The combination of genetic and environmental influences also plays a large role in the fruition of ASD. Metabolic function influences the genetic disposition of ASD during pregnancy. Metabolic function can be negatively influenced with the additional stress of diabetes, hypertension and obesity [11]. Krakowiak et al. investigated the metabolic conditions during pregnancy and the link to ASD, developmental impairments and developmental delays. Children born in California between the ages of 2-5 participated in the Childhood Autism Risks from Genetics and the Environment study, between January 2003 and June 2010. ASD, developmental impairments and/or developmental delays were assessed via a standardized test.
Information about the mother's health was collected from either medical records, or via an interview [11]. It was found that maternal metabolic function may be linked to neurodevelopmental problems appearing in children, as diabetic mothers of children with ASD scored lower on Mullen Scales of Early Learning. Results were compared to mothers without metabolic conditions. Although this is a small sampling, it may be helpful in developing a stronger understanding of maternal care [11].

In a case-control study from California, Volk et al. found a correlation between areas with high air pollution and increased risk factor for autism [12]. In areas with high air population, prenatal and early childhood exposure was negatively impacted. This includes exposure to traffic pollution during pregnancy and during the first year of life. Maternal infection during pregnancy has also been linked to autism [13]. Studies have linked a high rate of rheumatoid arthritis, type 1 diabetes, and celiac disease in mothers of children with ASD [14]. This may link autoimmune connections, or abnormal immune systems, as hereditary.

\section{Antipurinergic Therapy}

\section{Antipurinergic therapy: Poly(I:C) mouse model}

To assess the behavior of purinergic triggers in mice models of ASD, Naviaux et al. investigated the impact of APT with suramin on poly $(I: C)$ mouse models [2-6]. The aim of the study was to better understand the shared mechanisms of ASD and the mitochondrial pathways that influence the metabolic functions of non-infectious cellular stress. To do this, extracellular nucleotides (mitochondrial ATP) and APT were studied [2]. The study was conducted at the University of California, San Diego.

Purinergic triggers and behaviors where tested on the maternal immune activation mouse model (MIA) of ASD [3-5]. Mitochondrial ATP and ADP are part of a series of mitokines that function both inside and outside the cell. Outside the cell, ATP and ADP monitor purinergic receptors that live on the surface of every cell in the body [3-6]. P2X and P2Y are subsets of purinergic receptors that help regulate a spectrum of biological behaviors and mannerisms that impact known genetic dispositions of autism, including taste and senses related to taste [3-6], food allergies [3,7], proper food absorption and proper function of the gastrointestinal tract [2,15-17], and chronic inflammation [2].

The test was designed to understand if increased cellular danger response (CDR) helps manage the metabolic responses to cellular pathogens in MIA adult female mice that were exposed to a synthetic, double strand of poly $(I: C)$, at specific points during pregnancy [2]. Pregnant mice were injected with poly $(\mathrm{I}: \mathrm{C})$ to yield offspring with neurodevelopment traits shared in autism and schizophrenia [2]. MIA mouse models were used because of the high levels of cytokines in the fetal environment and fetal brain, which increase the risk of autism and schizophrenia in the offspring [2].

Pregnant mouse models exposed to doses of poly $(\mathrm{l}: \mathrm{C})$ at specific points during pregnancy imitate traditional pregnancy risks for offspring. In a study, pregnant mice susceptible to 
poly $(\mathrm{I}: \mathrm{C})$ at two different points during pregnancy (stage E12.5 and stage E17.5) found two different results: the first mouse model exhibited biochemical and metabolic dysfunctions and weakened behavioral and functioning abilities [2]; the second mouse model was shown to have intensified biochemical and metabolic dysfunctions that allowed for a stronger analysis of the traits and behaviors that resembled autism [2].

Accordingly, the biochemical and metabolic dysfunctions created by poly $(I: C)$ were solved with suramin treatment $[2,7]$. When MIA mouse models were attended with suramin, brain mitochondrial activity was lowered and oxygen consumption in the body was raised $[2,7]$. Body temperature was also stabilized $[2,7]$.

Normal activity and function blocks communication of cytokines that are influenced by innate immune activation [2]. As innate immunity is triggered by the maternal immune activation (MIA) mouse model, there is an anticipated reduction in quantity of cells in response to ATP. In the MIA mouse model, approximately $50 \%$ of synaptosomal FMRP was reduced and levels were stabilized by way of ATP $[2,6,18,19]$. The reduction of synaptosomal FMRP is evidence that the multisystem abnormalities existing in the MIA mouse model is reduced by way of ATP, despite mice having no genetic reductions of the Fragile $X(F m r 1)$ gene [2]. This finding proved consistent with the hypothesis that insufficient levels of FMRP is related to general CDR by way of hyperpurinergia in this specific view of ASD $[2,6,18,19]$.

In one study, APT in various ASD in the Fmr1 knockout mouse model was tested to determine if APT alleviates anxious social behavior, influences desires and inclinations in new situations, and synapse formation and metabolism $[6,18,19]$. Methodology included western analysis, behavioral analysis, electron microscopy, mass spectrometry and metabolomics $[6,19,20]$. Purinergic antagoniste suramin was administered at doses of 20 $\mathrm{mg} / \mathrm{kg}$ via weekly intraperitoneal injections, beginning at 9 weeks of age. Social actions and behavior improved, and metabolism and synaptosomal structure in the brain improved $[3-6,18,19]$. Specifically, the following irregularities were positively changed: amyloid $\beta$ precursor protein (APP), complement C1q, TDP43, endocannabinoid, purinergic synaptosomal glutamate, and IP3 receptor expression [4]. A review of the metabolic activity revealed 20 biochemical translations in accompany with improved symptoms, seventeen of which were found in conjunction with traits of ASD in humans. Eleven were in conjunction with the MIA mouse model. The described metabolic translations were also recognized as mediators that assisted the mechanics of preserved cell danger response (CDR) [3-6].

Although MIA and fragile $X$ mental retardation syndrome 1 mouse models of ASD have stark genetic and environmental determinants of ASD, suramin and APT treatment was found to reduce $50 \%$ of Fmr1 proteins (FMRP) and revive normal FMRP and normal behaviors in the MIA mouse model [6]. When FMRP activity is eliminated genetically, fragile $X$ syndrome develops [6].
In prior studies, the dynamic components and functions of purinergic signaling proved to be an appropriate test drug for MIA mouse models of autism [6]. Fragile $X$ mental retardation syndrome 1 was selected as a test because the irregularities in purinergic signaling may be the cause of environmental MIA and the genetic conditions that shape and impact fragile $X$ mental retardation syndrome 1 models [6].

Results concluded that APT aides in alleviating aspects of environmental MIA and genetic fragile $X$ mental retardation syndrome 1 models in ASD mouse models. Environmental and genetic irregularities are related to mitochondria and controlled by purinergic triggers [6].

In a different study by Naviaux et al. APT alleviated social behavior, anxiety during new experiences, and disruption to metabolic function in mouse models with ASD. Naviaux et al. used the MIA mouse model to determine the affect of controlled, single dose of suramin on social behavior and metabolic reaction of adult mice [20]. The MIA mouse model was administered with a controlled, single dose of suramin. Surmain helped repair social behavior, metabolism, and alleviate anxiety during new experiences. Purine metabolism was identified as the most significant regulatory pathway, determined through a metabolomic review. Naviaux et al. reported that 17 out of 18 broken metabolic were normalized by way of purine metabolism [20]. Two days following the suramin treatment, the level of suramin mixture in the plasma and brainstem was $7.64 \mu \mathrm{m}$ [20]. Five weeks following the experiment and post drug washout, the positive results seen via APT diminished. Naviaux et al. concluded that purine metabolism had a significant role in the behavior and metabolism of MIA mouse model, and a controlled, single dose of APT with suramin assists in alleviating metabolic and behavioral irregularities [20]. Metabolism influences neurotransmission and synaptic plasticity, which can cause symptoms of neurodevelopment disorders, including ASD [20]. This is especially significant during the onset or progression of infection, such as fever, during pregnancy. Metabolic changes that are caused due to infection increase the risk of ASD [20].

MIA mouse models exemplify symptoms of ASD and making pregnant mice vulnerable to poly $(I: C)$ reveals symptoms of ASD in offspring by triggering metabolic response to cell danger response. Continued existence of cell danger response is a common feature of ASD and Naviaux et al. postulate that purinergic signaling helps stabilize cell danger response. To test the affect of purinergic signaling on cell danger response, Naviaux et al. conducted a metabolomic study on APT in MIA mouse models [20]. A single dose of suramin administered to six-month old mice amended over $90 \%$ of disturbances in metabolic pathways, as well as behavioral aberrations. This finding helped determine that purine metabolism and purinergic signaling helps stabilize metabolism and behavior in MIA mouse models with disorders like ASD [20].

Poly(I:C) was injected in pregnant mouse models (>99\% pure; $<1 \%$ mononucleotide content), respectively. Behavioral tests were conducted when mice were nine weeks old. Behavioral tests included: social approach, rotarod, light-dark box, and absence of normal behaviors produced by suramin. At 21 or 
26-27 weeks, mice were given suramin or saline and tests were conducted again. The study only included male mice.

Social approach testing was defined as percentage of time socializing with stranger mouse compared to Lego blocks during the first 5 minutes of exposure time. Social approach was observed during 2-4 days, and following 5 weeks of suramin injection [20]. Social approach was defined as the time spent exploring chambers of the three-chamber box. During pilot experiments, mice spent most of their time exploring the first half of each block and social preference was calculated based on the social interactions (sniffing) of the mouse during the first 5 minutes of each phase [20]. Stranger mice were exposed to a wire cup during phase I for at least 30 minutes, respectively [20]. When the test mouse was removed from the chamber in phase II and replaced with an unfamiliar mouse, the unfamiliar mouse was set under one of the wire cups, with Lego blocks. At this time, the test mouse was again placed back in the box and allotted 10 minutes of explore time [20]. Explore time through the chambers, and sniff time, was recorded and scored. Saline and poly $(\mathrm{I}: \mathrm{C})$ mice groups were in equal number and based on their social approach scores when mice were 2.25 months old before controlled single-dose with saline, or 6.5 months old with suramin [20].

It was found mice groups exposed to saline and poly $(\mathrm{I}: \mathrm{C})$ scored the same as single dose of saline or suramin before the Tmaze [20]. T-maze tests determined comfort level with newness by testing how mice reacted to impromptu changes in a maze of black plexiglass. The test was designed according to Frye and Walf [21]. Reaction time and decision time was recorded. Alternated choices were observed at day 2 and day 4 , and following 5 weeks of suramin administration.

The rotarod test, adapted by Pallier et al., identified sensorimotor coordination and balance [22]. Mice were allotted 3 opportunities and 30 minutes of break time before another 3 opportunities were granted. With 2 periods, all mice were able to hold balance for 30 seconds. When rotarod time was increased, reaction time was recorded in seconds. The mice began at $4 \mathrm{rpm}$ and advanced to $40 \mathrm{rpm}$ across 5 minutes [20].

Light-dark box test determined how mice reacted to the change in light and dark and how anxiety affected behavior. The box was divided with a light chamber and a dark chamber and met with a door on either side. Mice were exposed to the light chamber and observed for 10 minutes. Time in the light chamber was observed and recorded [22-25].

Absence of abnormal behavior was conducted on non-MIA control animals; these animals were administered with saline as adults. Male mice were used for the studies. Data analysis was conducted using Student's t-test to compare suramin and saline treatment groups.

\section{FVB and Fragile X (Fmr1) knockout mouse model}

To better determine how APT will positively benefit metabolism, behavior, synaptic irregularities as pertaining to mouse models with fragile $X$ syndrome, the genetic loss of the fragile $X$ protein mouse strains of FVB were used to assess the impact of the Fragile $X$ (Fmr1) knockout on the said strain [6].
Mouse behavior was observed and recorded after mice were administered APT with suramin every week for one month. When mice were 13 weeks old, behavioral assessments were conducted. Behavioral tests were described as repulse inhibition mazes, reactions according to spontaneous noise to evoke a startling sensation, marble burying, sensorimotor function, Tmaze and social approach [6].

Using mass spectrometry, 60 pathways with 673 metabolites were assessed via partial least squares discriminate analysis (PLSDA), depicted visually and arranged in numerical order based on metabolic changes according to variable importance in projection (VIP) scores [4]. The small sample size found that suramin was responsible for $30 \%$ (20/60 pathways) of the pharmacometabolomic alterations in the aforementioned pathways [6].

Fragile $X$ protein knockout was positive. Distinct social conducts and behaviors are significant traits that resemble autism, as observed in mouse models with ASD [6]. When mouse models (Fmr1 null males) were given the opportunity to interact with a "stranger mouse" or an "inanimate object," mice were $26 \%$ less likely to spend time with a "stranger mouse" and $35 \%$ of mice experienced a "reduction in social novelty" [6], as determined by time socializing with a new mouse vs. an acquainted mouse [6]. The aforementioned traits that resemble autism in humans were tested with antipurinergic therapy with suramin in mouse models with ASD [6].

\section{ASD}

The main finding of the clinical studies was to advance therapies for purinergic signaling that cause ASD and other neurodevelopmental conditions. The authors noted that antipurinergic therapy provides new research opportunities into pathogenesis and new drug development for human ASD and other neurodevelopmental disorders [2]. Clinical studies focused on the purinergic signaling activities of mouse models with ASD to determine the abnormalities associated with autism to suggest alternative treatments.

APT with suramin was found to positively influence the irregularities in the structure of synapses, metabolism and behavior of fragile $X$ knockout mouse models [6]. APT with suramin also showed positive results in environmental MIA model and recent studies have shown positive results in genetic model of fragile $X$ knockout [6]. APT with suramin created the opportunity for cells to stabilize metabolism and revive cell activity [2].

APT with suramin can serve as a new drug to correct the social and behavioral traits similar in MIA models that resemble traits similar to humans with autism [2]. Limitations of the study reflect the range of genetic and environmental causes and influences of ASD. ASD is also translated differently from person to person; meaning, genetic and environmental influences are translated differently from person to person, although common traits exist across the spectrum. Although a number of strains were tested on mouse models, animal models are not ideal test subjects. With additional clinical trials and research, the 
properties of suramin and antipurinergic therapy with suramin may be a solution for neurodevelopmental disorders.

Suramin treatments for autistic children are not FDAapproved. Long-term therapy with suramin is not recommended as it may cause harmful side effects.

\section{Conclusions}

Results by Naviaux et al. concluded the medical conditions of ASD as produced by metabolic influences and caused by conserved cell danger response, respectively [2]. APT with suramin allowed cells to stabilize metabolism and revive cell activity [2]. Mitochondrial ATP, known as mitokines, trigger molecules made in the mitochondria, and exchange information about the health of neighboring cells and possible cell danger by way of purinergic signaling. The study determined APT as a corrective mechanism for 16 irregularities that characterized ASD and respective phenotypes [2]. This included: ERK1/2 and CAMKII signal transduction abnormalities, P2Y2 and P2X7 purinergic receptor expression, correction of the hypothermia, metabolic, mitochondrial, correction of social and locomotor dysfunctions, blockage of cerebellar Purkinje cell loss and preserving the correct form of ultrastructural synaptic dysmorphology [2].

Purinergic signaling abnormalities are of most significance in fragile $X$ syndrome, as they ranked the highest by the multivariate analysis, and are made up of about $20 \%$ of the highest numerical value of lipid metabolities [6]. The high activity of purinergic signaling and irregularities are also associated with behaviors and actions resembling autism in humans. Naviaux et al. note that the "inborn errors" located in purine, and pyrimidine metabolism, are characteristics of autism [6]. These cellular irregularities may cause hyperuricosuria, which has been linked to approximately $20 \%$ of children who do not have syndromic autism [6]. In 2012 and 2013, the association between purinergic signaling and purine metabolism in ASD was tested in MIA mouse models $[2,6]$. Recently, brain purinergic signaling was recognized as the most significant gene activity translation that is linked to abnormal behaviors in autistic children [6].

When Naviaux et al. used APT and suramin in the mouse model with fragile $X$, positive results were yielded for 17 of the 54 proteins that were classified as cerebral synaptosomes of Fmr1 knockout mice. Suramin lowered PI3 Kinase activity in the synaptosomal PI3/AKT/GSK3 $\beta$ pathway, lowered AKT, and raised the blockage phosphorylation of PI3K/AKT pathway protein glycogen synthase kinase $3 \beta$ (pGSK3 $\beta$ Ser9) approximately $47 \%$ $[6,19,20]$. Suramin also raised p70 S6 kinase Phosphorylation (pS6KThr389), a blocker of insulin receptor substrate 1 (IRS1), $46 \%[6,18,19]$. There were no alterations in mTOR activity or function and phosphorylation in cerebral synaptosomes in the mouse model of fragile $X$ were also left unchanged $[6,18,19]$.

Autistic children and adults with fragile $X$ syndrome have common brain abnormalities in the cerebellum, specifically the cerebellar vermis, which impact lobule VII. It was found that the Purkinje cells that were sustained during antipurinergic therapy at week 16 in the MIA model is determined by environmental influences and can be controlled by environmental influences [2]. Antipurinergic therapy reduced the amount of Purkinje cell loss during weeks 6 to $16[2,6]$. The synaptosomal purinergic receptor response of control animals (non-MIA mouse models) administered with suramin was also observed. It was found that "down regulation" did not cause any irregular behavior or behavioral traits in mice. More studies must be done on purinergic signaling to understand why antipurinergic therapy had no impact on behavior in healthy mice and had the opposite biochemical impact in healthy mice and mice with autistic behavioral traits $[2,6]$.

In the reviewed fragile $X$ mouse model test, APT with suramin positively influenced the irregularities in structure of synapses, metabolism and behavior. APT with suramin also showed positive results in environmental MIA model and recent studies have shown positive results in genetic model of fragile $X$ knockout. APT with suramin has the capacity to repair irregularities presented in fragile $X$ and MIA models because of the unique location near a foundational vehicle, related to mitochondria and controlled by purinergic signaling. This impacts both the progression and performance in both environmental MIA and genetic fragile $X[2,6]$.

Fmr1 knockout mice used in this study had post-synaptic masses that were hypomorphic. Cerebral synaptosomes that resembled standard appearance and standard post-synaptic densities were evident in mouse models treated with suramin [6]. In other studies by Naviaux et al. with MIA mouse models, MIA mouse models treated with APT had post-synaptic masses that were hypomorphic and unsteady. APT was also heavily populated in a specific area [6]. APT fixed the irregularities seen in the synaptic structure [6].

Behavioral traits were observed in the Fmr1 knockout model and compared to a control FVB model. The mice models, however, were not of the same litter and differences in action and etiquette (behavior) may be a result of differences in genotype and breed [6]. The focal point of the test was to determine how APT with suramin would influence and amend the irregularities in behavior, metabolism and synaptic organization. While other treatments have been assessed by distinguishing the synaptic irregularities and/or neurotransmitters that translate into various conditions of fragile $X$ in mice, the reviewed study found that APT with suramin plays the same function as the following, as included by Naviaux et al.: metabolic therapies, blockage of metabolic control enzyme glycogen synthase kinase $3 \beta$ (GSK3 $\beta$ ), drug inhibition of glutamatergic signaling with mGluR5 inhibitors, inhibition of endocannabinoid signaling, and genetic inhibition of amyloid $\beta$ precursor protein (APP) [6].

\section{Author's Contributions}

Dr. Rafie Hamidpour: Conception; analysis and interpretation; critical revision of the article; final approval of version to be published.

Dr. Soheila Hamidpour: Analysis and interpretation; critical revision of the article; final approval of version to be published. 
Dr. Mohsen Hamidpour: Analysis and interpretation; critical revision of the article; final approval of version to be published.

Marriam Zarabi: Acquisition of data; analysis and interpretation; drafting and critical revision of the article

Mahnaz Sohraby: Acquisition of data

Mina Shalari: Acquisition of data

\section{Acknowledgments}

Dr. Rafie Hamidpour, Department of Herbal Medicine, Pars Bioscience, Leawood, Kansas, United States

Dr. Soheila Hamidpour, School of Medicine Department of Pathology, University of Kansas City, Missouri, United States

Dr. Mohsen Hamidpour, Department of Hematology and Blood banking- Faculty of Paramedical Sciences- Shahid Beheshti University of Medical Sciences-Tehran-Iran

Marriam Zarabi, Pars Bioscience, Leawood, Kansas, United States

Mahnaz Sohraby, Pars Bioscience, Leawood, Kansas, United States

Mina Shalari, Pars Bioscience, Leawood, Kansas, United States

\section{References}

1. Developmental Disabilities Monitoring Network Surveillance Year Principal I, Centers for Disease C, Prevention. Prevalence of autism spectrum disorder among children aged 8 years - autism and developmental disabilities monitoring network, 11 sites, United States, 2010. MWR Surveill Summ 63: 1-21.

2. Naviaux RK, Zolkipli Z, Wang L, Nakayama T, Naviaux JC, et al. (2013) Antipurinergic therapy corrects the autism-like features in the poly(IC) mouse model. PLoS One 8: e57380.

3. Surprenant A, North RA (2009) Signaling at purinergic P2X receptors. Annu Rev Physiol 71: 333-359.

4. Leng Y, Yamamoto T, Kadowaki M (2008) Alteration of cholinergic, purinergic and sensory neurotransmission in the mouse colon of food allergy model. Neurosci Lett 445: 195-198.

5. Abbracchio MP, Burnstock G, Verkhratsky A, Zimmermann $H$ (2009) Purinergic signalling in the nervous system: an overview. Trends Neurosci 32: 19-29.

6. Naviaux JC, Wang L, Li K, Bright AT, Alaynick WA, et al. (2015) Antipurinergic therapy corrects the autism-like features in the Fragile X (Fmr1 knockout) mouse model. Mol Autism 6: 1 .

7. Lao J (2014) Autism Spectrum Disorders: An Intervention Approach Based on Genomic Analysis Biol Med. S1 002: 2-12.

8. Anney R, Klei L, Pinto D, Almeida J, Bacchelli E, et al. (2012) Individual common variants exert weak effects on the risk for autism spectrum disorderspi. Hum Mol Genet 21: 4781-4792.

9. Pinto D, Pagnamenta AT, Klei L, Anney R, Merico D, et al. (2010) Functional impact of global rare copy number variation in autism spectrum disorders. Nature 466: 368-372.
10. Casey JP, Magalhaes T, Conroy JM, Regan R, Shah N, et al. (2012) A novel approach of homozygous haplotype sharing identifies candidate genes in autism spectrum disorder. Hum Genet 131: 565-579.

11. Krakowiak P, Walker CK, Bremer AA, Baker AS, Ozonoff S, et al. (2012) Maternal metabolic conditions and risk for autism and other neurodevelopmental disorders. Pediatrics 129: e1121e1128.

12. Volk HE, Lurmann F, Penfold B, Hertz-Picciotto I, McConnell R (2013) Traffic-related air pollution, particulate matter, and autism. JAMA Psychiatry 70: 71-77.

13. Patterson PH (2011) Maternal infection and immune involvement in autism. Trends Mol Med 17: 389-394.

14. Atladóttir HO, Pedersen MG, Thorsen P, Mortensen PB, Deleuran $B$, et al. (2009) Association of family history of autoimmune diseases and autism spectrum disorders. Pediatrics 124: 687-694.

15. Careaga M, Van de Water J, Ashwood P (2010) Immune dysfunction in autism: a pathway to treatment. Neurotherapeutics 7: 283-292.

16. Gallego D, Vanden P, Farre R, Tack J, Jimenez M (2008) P2Y1 receptors mediate inhibitory neuromuscular transmission and enteric neuronal activation in small intestine. Neurogastroenterol Motil 20: 159-68.

17. Matos JE, Sorensen MV, Geyti CS, Robaye B, Boeynaems JM, et al. (2007) Distal colonic $\mathrm{Na}(+)$ absorption inhibited by luminal P2Y(2) receptors. Pflugers Arch 454: 977-987.

18. McGeary RP, Bennett AJ, Tran QB, Cosgrove KL, Ross BP (2008) Suramin: clinical uses and structure-activity relationships. Mini Rev Med Chem 8: 1384-1394.

19. Brigotti M, Alfieri RR, Petronini PG, Carnicelli D (2006) Inhibition by suramin of protein synthesis in vitro. Ribosomes as the target of the drug. Biochimie 88: 497-503.

20. Naviaux JC, Schuchbauer MA, Li K, Wang L, Risbrough VB, et al. (2014) Reversal of autism-like behaviors and metabolism in adult mice with single-dose antipurinergic therapy. Transl Psychiatry 4: e400.

21. Frye CA, Walf AA (2008) Effects of progesterone administration and APPswe+PSEN1Deltae9 mutation for cognitive performance of mid-aged mice. Neurobiol Learn Mem 89: 17-26.

22. Pallier PN, Drew CJ, Morton AJ (2009) The detection and measurement of locomotor deficits in a transgenic mouse model of Huntington's disease are task- and protocol-dependent: influence of non-motor factors on locomotor function. Brain research bulletin 78: 347-355.

23. Folkman J, Shing $Y$ (1992) Angiogenesis. J Biol Chem 267: 10931-10934.

24. Teicher B, Sotomayor E, Huang Z (1992) Antiangiogenic agents potentiate cytotoxic cancer therapies against primary and metastatic disease. Cancer Res 52: 6702-6704.

25. Gradishar WJ, Soff G, Liu J, Cisneros A, French S, et al. (2000) A pilot trial of suramin in metastatic breast cancer to assess antiangiogenic activity in individual patients. Oncology 58: 324-333. 